\title{
USING SYMBOLIC COMPUTATION IN THE CHARACTERIZATION OF FRICTIONAL INSTABILITIES INVOLVING ORTHOTROPIC MATERIALS
}

\author{
Mohamed A. AGWA ${ }^{a}$, António PINTO dA COSTA ${ }^{b, *}$ \\ ${ }^{a}$ Department of Mechanical Design and Production Engineering \\ Zagazig University, P.O. Box 44519, Zagazig, Egypt \\ e-mail: mwa.agwa@gmail.com \\ ${ }^{b}$ Department of Civil Engineering, Architecture and Georesources / ICIST
University of Lisbon, Avenida Rovisco Pais, 1049-001 Lisbon, Portugal
e-mail: antonio.pinto.da.costa@tecnico.ulisboa.pt
}

The present work addresses the problem of determining under what conditions the impending slip state or the steady sliding of a linear elastic orthotropic layer or half space with respect to a rigid flat obstacle is dynamically unstable. In other words, we search the conditions for the occurrence of smooth exponentially growing dynamic solutions with perturbed initial conditions arbitrarily close to the steady sliding state, taking the system away from the equilibrium state or the steady sliding state. Previously authors have shown that a linear elastic isotropic half space compressed against and sliding with respect to a rigid flat surface may get unstable by flutter when the coefficient of friction $\mu$ and Poisson's ratio $\nu$ are sufficiently large. In the isotropic case they have been able to derive closed form analytic expressions for the exponentially growing unstable solutions as well as for the borders of the stability regions in the space of parameters, because in the isotropic case there are only two dimensionless parameters $(\mu$ and $\nu$ ). Already for the simplest version of orthotropy (an orthotropic transversally isotropic material) there are seven governing parameters ( $\mu$, five independent material constants and the orientation of the principal directions of orthotropy) and the expressions become very lengthy and literally impossible to manipulate manually. The orthotropic case addressed here is impossible to solve with simple closed form expressions, and therefore the use of computer algebra software is required, the main commands being indicated in the text.

Keywords: Coulomb friction, dynamic instabilities, surface solutions, orthotropic material.

\section{Introduction}

The aim of this work is to explore automatic symbolic computation in the solution of two related problems of engineering mechanics. We deal with elastic bodies with a flat frictional interface - a layer of finite depth and a half space-that, under certain circumstances, may exhibit unstable behaviours, i.e., the tendency to respond with exponentially growing displacements and reactions.

The appropriate combinations of stiffness, mass and friction under which friction-induced instabilities may occur are computed by the search of smooth dynamic solutions, beginning arbitrarily close to an equilibrium state or to a steady sliding state, which take the system away from equilibrium in an exponential oscillatory

\footnotetext{
*Corresponding author
}

(flutter) or non-oscillatory (divergence) manner. These two types of dynamic instability of non-isotropic solids in the presence of a frictional interface are investigated with analytic models with a strong component of symbolic manipulation. Closed form expressions for the coefficient of friction at the onset of instability are derived by widely exploring automatic symbolic computation resources.

It was only twenty years ago that Martins et al. (1992; 1995) and Adams (1995) derived the first conditions for the occurrence of frictional instabilities in elastic spaces. Ibrahim (1994) as well as Martins and Raous (2002) are exclusively dedicated to the conditions for the occurrence of frictional instabilities or to their effects. The first attempts to model instabilities in nonisotropic solids were made by Agwa and Pinto da Costa (2008; 2011), as well as Pinto da Costa and 
Agwa (2009), the present study being a continuation of that effort. Here we consider orthotropic transversely isotropic linear elastic materials with principal directions of orthotropy that are, in general, skewed with respect to the direction of sliding. As we will see, it is this skewness and the larger number of material parameters that enforce the intervention of symbolic manipulation, without which the problems treated in this study would hardly be solved. The intensive use of symbolic computation in this study enabled the derivation of new closed form expressions for the critical coefficients of friction and the derivation of (very lengthy) analytical expressions eventually used to obtain new numerical results summarized in graphs.

The formulas and procedures included in this study may be useful in the design of orthotropic composite materials operating under unilateral frictional contact conditions. A natural extension of the present work is the application of its results to the design of the conditions in industrial environments to which orthotropic composite materials may be submitted in order to preclude frictional contact instabilities.

\section{Surface divergence instability}

A deformable material occupies a region in the $(x, y, z)$ plane defined to be unbounded in the $x$ direction, to be limited to the interval $[0, h]$ in the $y$ direction and to have a certain uniform thickness in the $z$ direction (Fig. 1). The material particles at $y=h$ are fixed while the

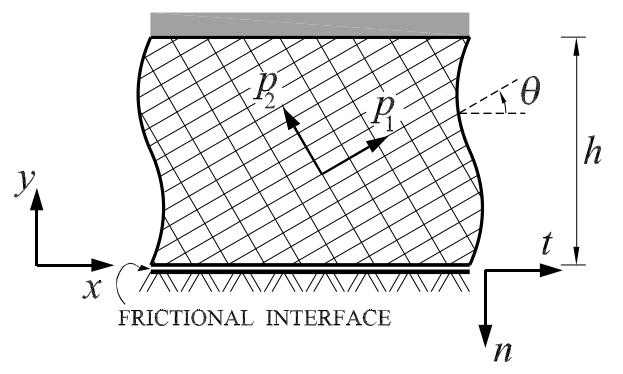

Fig. 1. Elastic orthotropic infinite layer in frictional contact with a flat surface.

particles at $y=0$ are kinematically constrained so as not to abandon the region $y \geq 0$ and statically constrained to follow Coulomb's friction law $\sigma_{y x} \in \mu \sigma_{y y} \operatorname{Sign}(S-$ $\dot{u})$ in the direction tangent to the frictional boundary. Derivation with respect to the time variable $t$ is denoted by the dot over the corresponding variable. The possibly nonvanishing velocity of the flat rough surface in the tangent direction $t$ is denoted by $S$ (positive to the right), $\sigma_{y x}$ and $\sigma_{y y}$ are the usual components of the Cauchy stress tensor, $\mu \geq 0$ stands for the constant coefficient of friction, the Cartesian components of the displacement field are $u$ ( $x$ component $), v(y$ component $)$ and $w(z$ component), and $\operatorname{Sign}(z)$ is the multi-mapping yielding 1 for $z>0$, the interval $[-1,1]$ for $z=0$, and -1 for $z<0$. The skew angle of the principal directions of orthotropy $p_{1}$ and $p_{2}$ respectively with respect to $x$ and $y$ is denoted by $\theta$. The components of the stress tensor organized as a vector

$$
\boldsymbol{\sigma}=\left\{\sigma_{x x}, \sigma_{y y}, \sigma_{x y}, \sigma_{z z}, \sigma_{y z}, \sigma_{x z}\right\}^{\mathrm{T}}
$$

may be expressed in terms of the components of the strain tensor

$$
\varepsilon=\left\{u_{, x}, v_{, y}, u_{, y}+v_{, x}, w_{, z}, v_{, z}+w_{, y}, u_{, z}+w_{, x}\right\}^{\mathrm{T}}
$$

by premultiplying $\varepsilon$ by a stiffness matrix with components $D_{i j}$ that are functions of (i) the elastic parameters $E_{1}$, $E_{2}, G_{12}, \nu_{12}, \nu$ of an orthotropic transversely isotropic material defined with respect to the principal directions of orthotropy and (ii) of the skew angle $\theta$ (Batoz and Dhatt, 1990; Rand and Rovenski, 2005; Agwa and Pinto da Costa, 2008). The coefficients $D_{i j}$ depend on whether the layer is in a state of plane strain or in a state of plane stress.

Assuming that the particles at the bottom are in persistent contact in impending slip to the left with respect to the rough surface, we seek exponentially growing nonoscillatory solutions $u(y, t)=\exp (\lambda t) U(y)$ and $v(y, t)=\exp (\lambda t) V(y)$ with $\lambda>0$ of the problem consisting in the conjunction of the following conditions:

momentum equations: $\sigma_{x x, x}+\sigma_{y x, y}=\varrho \ddot{u}$ and $\sigma_{x y, x}+$ $\sigma_{y y, y}=\varrho \ddot{v}$ ( $\varrho$ is the material's volumetric mass, assumed uniform);

constitutive equations: $\sigma_{x x}=D_{11} u_{, x}+D_{12} v_{, y}+$ $D_{13}\left(u_{, y}+v_{, x}\right), \sigma_{y y}=D_{12} u_{, x}+D_{22} v_{, y}+D_{23}\left(u_{, y}+\right.$ $\left.v_{, x}\right)$ and $\sigma_{x y}=D_{13} u_{, x}+D_{23} v_{, y}+D_{33}\left(u_{, y}+v_{, x}\right)$ $\left(()_{, \alpha}\right.$ stands for the derivative of ( ) with respect to $\alpha ; D_{i j}$ are elements of the symmetric stiffness matrix connecting the elements of the stress and deformation tensors, written in terms of the $x$ and $y$ directions);

kinematic boundary conditions: $u(x, h)=v(x, h) \equiv$ 0 and $v(x, 0) \equiv 0$;

frictional boundary conditions: $\sigma_{y x}=\mu \sigma_{y y}$.

The conjugation of the assumed variable separated solution with the constitutive and momentum equations yields

$$
\left[\begin{array}{ll}
D_{33} & D_{23} \\
D_{23} & D_{22}
\end{array}\right]\left\{\begin{array}{l}
U^{\prime \prime}(y) \\
V^{\prime \prime}(y)
\end{array}\right\}=\varrho \lambda^{2}\left\{\begin{array}{l}
U(y) \\
V(y)
\end{array}\right\},
$$

with ()$^{\prime}$ denoting derivation with respect to $y$. The general solution to (1), sought with the Maple (2013) 
command dsolve with the option symbolic, corresponds to

$$
\{u(y, t)\}=\left\{\begin{array}{c}
\frac{D_{23} S_{1}^{2}}{\rho-D_{33} S_{1}^{2}}\left[C_{1} \exp \left(S_{1} \lambda y\right)\right. \\
\left.+C_{2} \exp \left(-S_{1} \lambda y\right)\right] \\
+\frac{D_{23} S_{2}^{2}}{\rho-D_{33} S_{2}^{2}}\left[C_{3} \exp \left(S_{2} \lambda y\right)\right. \\
\left.+C_{4} \exp \left(-S_{2} \lambda y\right)\right] \\
\\
C_{1} \exp \left(S_{1} \lambda y\right) \\
+C_{2} \exp \left(-S_{1} \lambda y\right) \\
+C_{3} \exp \left(S_{2} \lambda y\right) \\
+C_{4} \exp \left(-S_{2} \lambda y\right)
\end{array}\right\} \exp (\lambda t)
$$

where $C_{1}$ to $C_{4}$ are real integration constants. When $D_{22} D_{33}-D_{23}^{2} \neq 0$ is assumed, we get

$$
S_{1 \text { or } 2}=\left[\rho \frac{D_{22}+D_{33} \pm \sqrt{\Delta}}{2\left(D_{22} D_{33}-D_{23}^{2}\right)}\right]^{\frac{1}{2}}
$$

with $\Delta=\left(D_{22}+D_{33}\right)^{2}-4\left(D_{22} D_{33}-D_{23}^{2}\right)$, and the coefficient of friction as a function of the (exponential) rate growth in time is given by the expression

$$
\mu=\frac{A}{B}
$$

where

$$
\begin{aligned}
A= & S_{1} \operatorname{coth}\left(S_{1} \lambda h\right)\left(D_{23}+a_{1} D_{33}\right) \\
& -S_{2} \operatorname{coth}\left(S_{2} \lambda h\right)\left(D_{23}+a_{2} D_{33}\right), \\
B= & S_{1} \operatorname{coth}\left(S_{1} \lambda h\right)\left(D_{22}+a_{1} D_{23}\right) \\
& -S_{2} \operatorname{coth}\left(S_{2} \lambda h\right)\left(D_{22}+a_{2} D_{23}\right), \\
a_{1}= & \frac{D_{23} S_{1}^{2}}{\varrho-D_{33} S_{1}^{2}}, \quad a_{2}=\frac{D_{23} S_{2}^{2}}{\varrho-D_{33} S_{2}^{2}}
\end{aligned}
$$

and coth denotes the hyperbolic cotangent function. For a more complete list of Maple commands, refer to Table A1 in Appendix.

For states of plane strain, Fig. 2 presents the evolution of the eigenvalue $\lambda$ as a function of the coefficient of friction for a fixed set of parameters $E_{2}$, $G_{12}, \nu_{12}$ and $\nu$; several values of $E_{1}$ increasing from plot (a) to plot (e) were considered and, in each plot, several orientations of the principal directions of orthotropy. It can be seen from Fig. 2 that all the curves have vertical tangents at the onset of instability (vanishing rate of growth of the solution, i.e., $\lambda=0$ ), something that can only be proven by the symbolic derivation of the expression (4) of $\mu$ with respect to $\lambda$ and then by the evaluation of the resulting expression at $\lambda=0$. In terms of Maple commands,

dmu ( lambda) :=diff (mu ( lambda) , lambda), and then

limit (dmu ( lambda), lambda=0 ), which yields zero, proving the vertical slopes for vanishing $\lambda$.

Note that, for the assumed state of impending slip to the left with respect to the obstacle, large ratios $E_{1} / E_{2}$ (increasing from Figs. 22(a) to 2 (e)) and angles $\theta$ approaching $90^{\circ}$ from below are favourable conditions for the onset of divergence instability to occur at low values of the coefficient of friction: the intersection of the curves with the $\mu$ axis occurs for lower values of $\mu$.

Explicit expressions for the coefficients of friction $\mu_{\mathrm{cr}}$ at the onset of surface divergence instability may easily be obtained from (4) by computing symbolically the limit as $\lambda \rightarrow 0$. Using the notation $s=\sin \theta$ and $c=\cos \theta$, for states of plane stress, the critical coefficient of friction at the onset of divergence instability is

$$
\mu_{\mathrm{cr}}\left(\frac{E_{1}}{E_{2}}, \frac{G_{12}}{E_{2}}, \nu_{12}, \theta\right)=\frac{f\left(E_{1} / E_{2}, G_{12} / E_{2}, \nu_{12}, \theta\right)}{g\left(E_{1} / E_{2}, G_{12} / E_{2}, \nu_{12}, \theta\right)},
$$

where

$$
\begin{aligned}
f\left(\frac{E_{1}}{E_{2}},\right. & \left.\frac{G_{12}}{E_{2}}, \nu_{12}, \theta\right) \\
= & \left(s^{2}-c^{2}\right)^{2}\left(\nu_{12}^{2}-\frac{E_{1}}{E_{2}}\right) \frac{G_{12}}{E_{2}} \\
& +\left(2 \nu_{12}-1-\frac{E_{1}}{E_{2}}\right) s^{2} c^{2} \frac{E_{1}}{E_{2}}
\end{aligned}
$$

and

$$
\begin{aligned}
g( & \left.\frac{E_{1}}{E_{2}}, \frac{G_{12}}{E_{2}}, \nu_{12}, \theta\right) \\
= & {\left[2\left(s^{2}-c^{2}\right)\left(\frac{E_{1}}{E_{2}}-\nu_{12}^{2}\right) \frac{G_{12}}{E_{2}}\right.} \\
& \left.-s^{2}\left(\frac{E_{1}}{E_{2}}\right)^{2}+\left(\left(s^{2}-c^{2}\right) \nu_{12}+c^{2}\right) \frac{E_{1}}{E_{2}}\right] s c,
\end{aligned}
$$

while for states of plane strain

$\mu_{\mathrm{cr}}\left(\frac{E_{1}}{E_{2}}, \frac{G_{12}}{E_{2}}, \nu_{12}, \nu, \theta\right)=\frac{f\left(E_{1} / E_{2}, G_{12} / E_{2}, \nu_{12}, \nu, \theta\right)}{g\left(E_{1} / E_{2}, G_{12} / E_{2}, \nu_{12}, \nu, \theta\right)}$,

where

$$
\begin{aligned}
f\left(\frac{E_{1}}{E_{2}}, \frac{G_{12}}{E_{2}}, \nu_{12}, \nu, \theta\right) \\
=s^{2} c^{2}\left[\left(1-\nu^{2}\right)\left(\frac{E_{1}}{E_{2}}\right)^{2}\right. \\
\left.-\left(2(1+\nu) \nu_{12}-1\right) \frac{E_{1}}{E_{2}}-\nu_{12}^{2}\right] \\
+\left(s^{2}-c^{2}\right)^{2} \frac{G_{12}}{E_{2}}\left[\left(1-\nu^{2}\right) \frac{E_{1}}{E_{2}}\right. \\
\left.-2(1+\nu) \nu_{12}^{2}\right]
\end{aligned}
$$




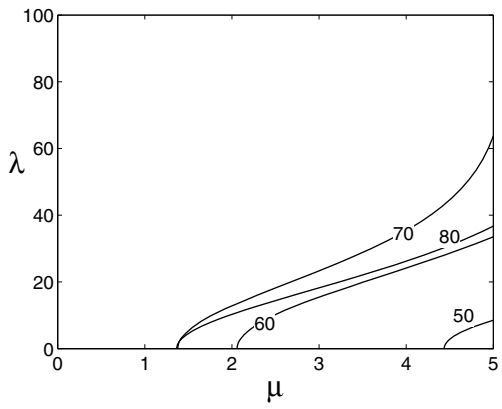

(a)

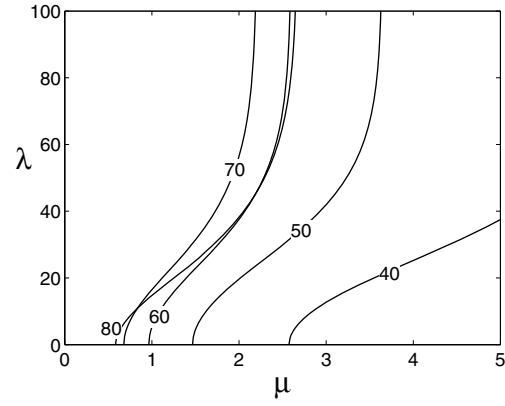

(b)

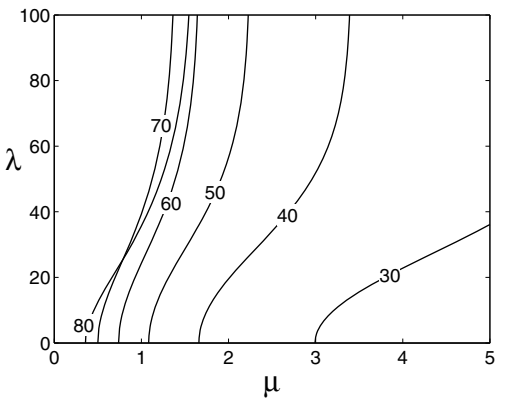

(c)

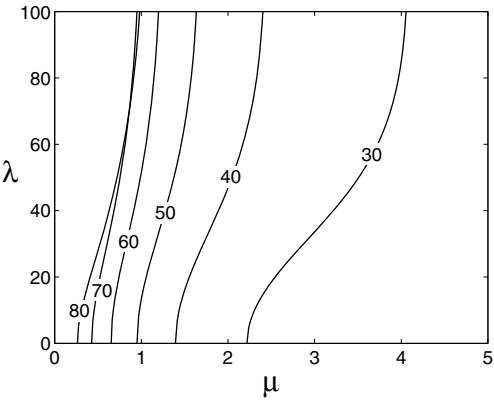

(d)

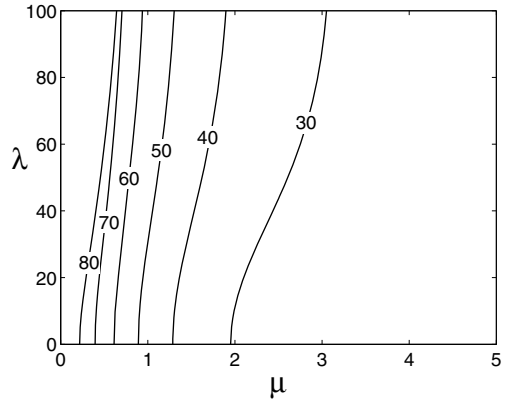

(e)

Fig. 2. Solution rate of the exponential growth $\lambda$ in terms of the coefficient of friction for $E_{2}=1, G_{12}=0.1, \nu_{12}=0.4, \nu=0.3$, and the angle $\theta$ (in degrees, near the curves) defining the orientation of the principal directions of orthotropy. State of plane strain: $E_{1}=1$ (a), $E_{1}=2$ (b), $E_{1}=4$ (c), $E_{1}=8$ (d) and $E_{1}=16$ (e).

and

$$
\begin{aligned}
g\left(\frac{E_{1}}{E_{2}},\right. & \left.\frac{G_{12}}{E_{2}}, \nu_{12}, \nu, \theta\right) \\
= & {\left[s c\left(c^{2}-s^{2}\right)(1+\nu) \nu_{12}-s c^{3}\right] \frac{E_{1}}{E_{2}} } \\
& +s c^{3} \nu_{12}^{2}+\left(1-\nu^{2}\right) s^{3} c\left(\frac{E_{1}}{E_{2}}\right)^{2} \\
& +s c\left(s^{2}-c^{2}\right) \frac{G_{12}}{E_{2}}\left[2\left(\nu^{2}-1\right) \frac{E_{1}}{E_{2}}\right. \\
& \left.+4(1+\nu) \nu_{12}^{2}\right]
\end{aligned}
$$

The plots in Fig. 3 illustrate how $\mu_{\text {cr }}$ decreases with an increase in $E_{1} / E_{2}$ with all the other parameters fixed. Smaller values of $G_{12} / E_{2}$ promote instability for $\theta$ approaching $90^{\circ}$ from below, while instability is promoted for larger values of $G_{12} / E_{2}$ when $\theta$ is small.

Another perspective may be taken with the set of plots in Fig. 4. Clearly, when the orientation of the principal direction of orthotropy $p_{1}$ approaches $90^{\circ}$ from below (and the layer is in impending slip to the left), the critical values of $\mu$ for divergence are lower-they may easily reach values much smaller than 1 . It is observed that for larger values of $E_{1}$, and for a fixed $\theta$, the range of $E_{2}$ leading to small values of $\mu_{\mathrm{cr}}$ increases.

\section{Surface flutter instability}

The next problem is associated with a different kind of instability-flutter instability, beginning at the frictional interface of an elastic (also orthotropic) half space (Fig. 5] with an exponentially growing oscillatory motion with respect to the steady sliding solution. The first results

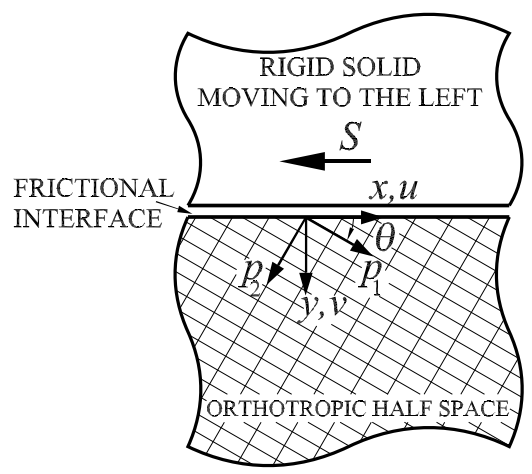

Fig. 5. Elastic orthotropic half space in frictional contact with a flat surface.

of frictional instabilities in half spaces presented by Martins et al. $(1992 ; 1995)$ were obtained with no help from symbolic computation. In the cited papers, the authors derived manually the conditions which completely 


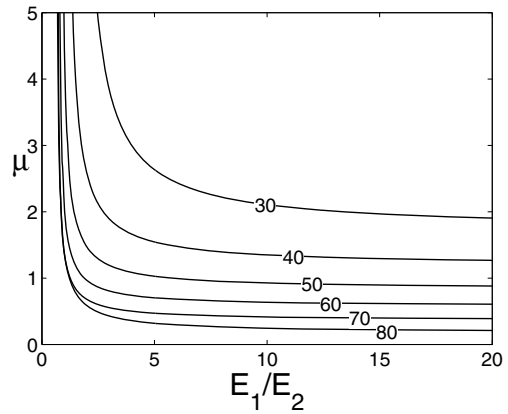

(a)

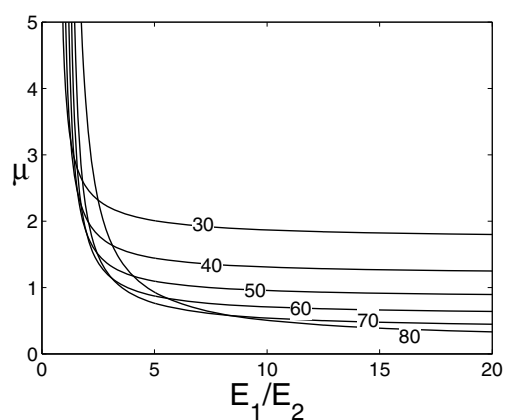

(c)

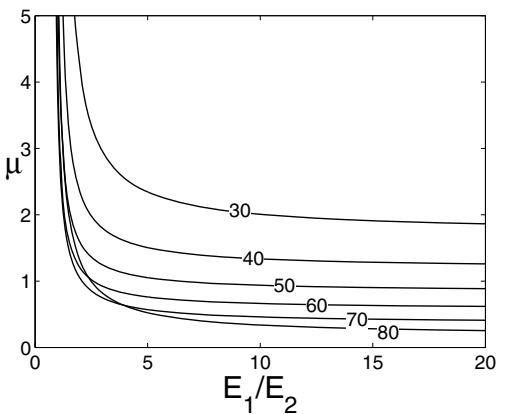

(b)

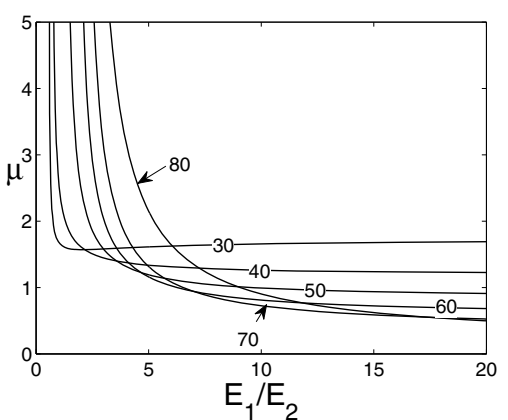

(d)

Fig. 3. Coefficient of friction at the onset of divergence instability as a function of $E_{1} / E_{2}$ for $\nu_{12}=0.4, \nu=0.3$, for four values of $G_{12} / E_{2}$, and several values of $\theta$ (indicated on the curves). State of plane strain: $G_{12} / E_{2}=0.1$ (a), $G_{12} / E_{2}=0.25$ (b), $G_{12} / E_{2}=0.5$ (c) and $G_{12} / E_{2}=1.0(\mathrm{~d})$.

characterize the situations of flutter instability in an isotropic half space in contact with a rough moving rigid surface. A similar procedure for orthotropic materials is out of question, as will be seen below. The steady sliding solution, whose instability conditions we wish to determine, is defined by $\sigma_{y y}^{s}(x, 0)<0, v^{s}(x, 0)=0$ (persistent contact) and $\sigma_{y x}^{s}(x, 0)+\mu \sigma_{y y}^{s}(x, 0)=0$ (persistent sliding) (see Martins et al., 1995). The upper rigid surface is sliding to the left with a nonvanishing velocity $S$ which is compatible with a shear stress $\sigma_{y x}^{s}(x, 0)=-\mu \sigma_{y y}^{s}(x, 0)>0$. In much the same way as in the previous section, the perturbed solution must comply with the following set of conditions:

momentum equations: $\sigma_{x x, x}+\sigma_{x y, y}=\varrho \ddot{u}$ and $\sigma_{x y, x}+$ $\sigma_{y y, y}=\varrho \ddot{v}$

constitutive equations: $\sigma_{x x}=D_{11} u_{, x}+D_{12} v_{, y}+$ $D_{13}\left(u_{, y}+v_{, x}\right), \sigma_{y y}=D_{12} u_{, x}+D_{22} v_{, y}+D_{23}\left(u_{, y}+\right.$ $\left.v_{, x}\right)$ and $\sigma_{x y}=D_{13} u_{, x}+D_{23} v_{, y}+D_{33}\left(u_{, y}+v_{, x}\right)$;

kinematic boundary conditions at $\boldsymbol{y}=\mathbf{0}: v(x, 0, t)=$ 0 ;

frictional boundary conditions at $\boldsymbol{y}=\mathbf{0}: \sigma_{y x}(x, 0, t)+$ $\mu \sigma_{y y}(x, 0, t)=0$.
We look for perturbed solutions of the type

$$
\left\{\begin{array}{l}
u(x, y, t) \\
v(x, y, t)
\end{array}\right\}=\left\{\begin{array}{l}
A \\
B
\end{array}\right\} e^{-b y} \exp (\mathrm{i} k(x-c t)),
$$

where $b$ may be a complex number provided its real part is positive (to ensure a displacement field exponentially decaying towards the interior of the elastic medium), i denotes the imaginary unit, $k>0$ is the wave number and $c$ is the complex wave velocity (note that, in this section, $c$ has nothing in common with the cosine of the previous section). For $\operatorname{Re}(c) \neq 0$ the solution oscillates and if, in addition, $\operatorname{Im}(c)>0$, it grows exponentially, too (a flutter instability).

The first step to build a perturbed solution in the neighbourhood of the steady sliding state consists in substituting (12) in the equations obtained by inserting the constitutive ones into the momentum equations, which leads to the following eigenvalue problem with a complex matrix:

$$
\left[\begin{array}{cc}
b^{2} D_{33} & b^{2} D_{23}-k^{2} D_{13} \\
+k^{2}\left(\varrho c^{2}-D_{11}\right) & -b k\left(D_{12}+D_{33}\right) \mathrm{i} \\
-2 b k D_{13} \mathrm{i} & \\
b^{2} D_{23}-k^{2} D_{13} & b^{2} D_{22} \\
-b k\left(D_{12}+D_{33}\right) \mathrm{i} & +k^{2}\left(\varrho c^{2}-D_{33}\right) \\
& -2 b k D_{23} \mathrm{i}
\end{array}\right]\left\{\begin{array}{l}
A \\
B
\end{array}\right\}
$$




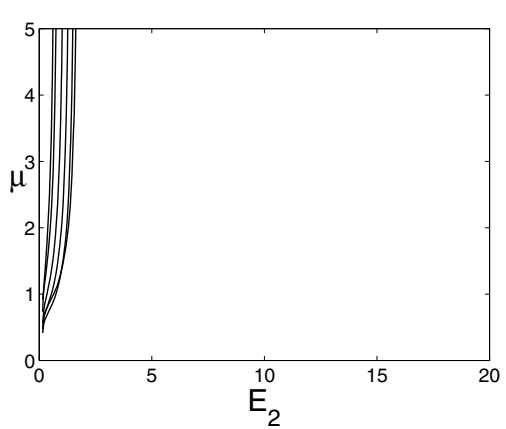

(a)

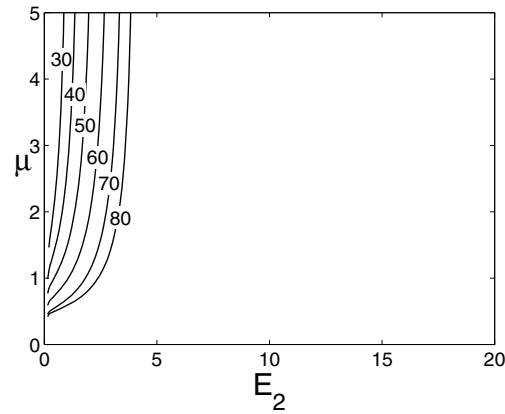

(b)

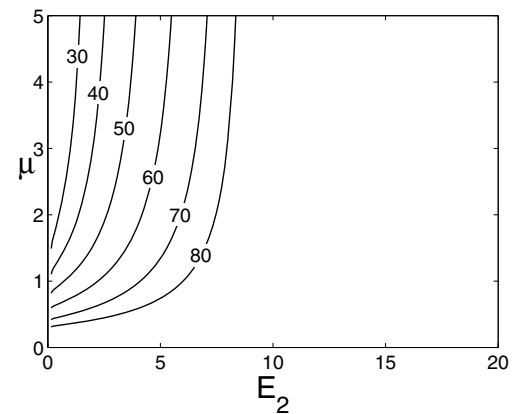

(c)

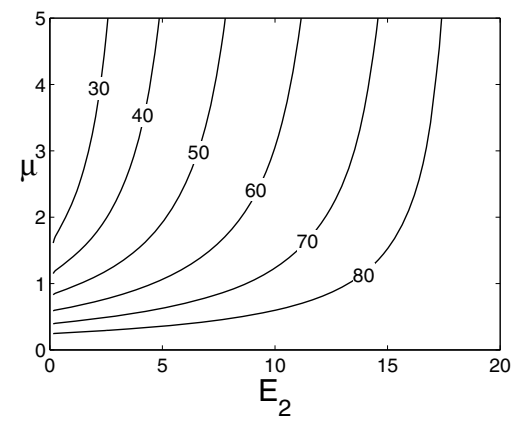

(d)

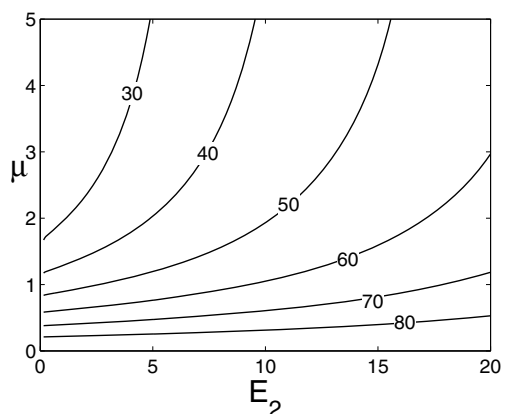

(e)

Fig. 4. Coefficient of friction at the onset of divergence instability as a function of $E_{2}$ for $\nu_{12}=0.4, \nu=0.3, G_{12} / E_{2}=0.1$, for several values of $E_{1}$ (one for each plot) and $\theta$ (indicated on the curves). The values of $\theta$ in (a) are the same as those of the other plots: from $30^{\circ}$ for the leftmost curve to $80^{\circ}$ for the rightmost curve. State of plane strain: $E_{1}=1$ (a), $E_{1}=2$ (b), $E_{1}=4$ (c), $E_{1}=8$ (d) and $E_{1}=16$ (e).

$$
=\left\{\begin{array}{l}
0 \\
0
\end{array}\right\},
$$

in which $b$ plays the role of the eigenvalue, for a fixed velocity $c$ and fixed constitutive properties. Under the assumption of a nonvanishing wave number $k$, the Maple command collect $(\operatorname{det}(\mathrm{M}), \mathrm{b})$ enables one to organize the characteristic equation of the coefficient matrix in (13) (denoted by $\mathrm{M}$ ) in terms of a complex polynomial of powers of $b / k$,

$$
\begin{aligned}
d_{1}\left(\frac{b}{k}\right)^{4} & +\left(d_{2}+d_{3} c^{2}\right)\left(\frac{b}{k}\right)^{2}+d_{4}+d_{5} c^{2}+d_{6} c^{4} \\
& +\mathrm{i} \frac{b}{k}\left[d_{7}\left(\frac{b}{k}\right)^{2}+d_{8}+d_{9} c^{2}\right]=0,
\end{aligned}
$$

where $d_{1}, \ldots, d_{9}$ depend on the material properties (see Agwa and Pinto da Costa, 2011). For a more complete list of Maple commands, refer to Table A2 in Appendix.

The four solutions $b / k$ are sought with solve and allvalues commands, from which we are only interested in the two with a positive real part. In the special cases for which coefficients $d_{7}, d_{8}$ and $d_{9}$ vanish (namely, isotropic materials), Eqn. (14) is a biquadratic equation that obviously does not require automatic symbolic manipulation; in the general orthotropic transversely isotropic materials, Eqn. (14) can nowadays be solved with a symbolic manipulator in order to express the solutions $b / k$ in terms of the wave velocity $c$. The two solutions $b^{\prime}$ and $b^{\prime \prime}$ with positive real parts are associated with two eigenvectors of (13), $\left(A^{\prime}, B^{\prime}\right)$ and $\left(A^{\prime \prime}, B^{\prime \prime}\right)$; the general solution is then obtained by the linear combination

$$
\begin{aligned}
& \left\{\begin{array}{l}
u(x, y, t) \\
v(x, y, t)
\end{array}\right\} \\
& =\left\{\begin{array}{l}
A^{\prime} e^{-b^{\prime} y}+A^{\prime \prime} e^{-b^{\prime \prime} y} \\
B^{\prime} e^{-b^{\prime} y}+B^{\prime \prime} e^{-b^{\prime \prime} y}
\end{array}\right\} \exp (\mathrm{i} k(x-c t)),
\end{aligned}
$$

in which the constants $A^{\prime}, B^{\prime}, A^{\prime \prime}$ and $B^{\prime \prime}$ are not independent because they have to satisfy the two possible ratios $(B / A)^{\prime}$ and $(B / A)^{\prime \prime}$ due to the linear dependence of the two equations in (13). Note that $b^{\prime}$ and $b^{\prime \prime}$, symbolically dependent on the velocity $c$, are in general very lengthy expressions that can only be manipulated in an automatic symbolic manner.

A second eigenvalue problem is obtained by enforcing the general solution (15) to satisfy the kinematic boundary conditions together with the frictional boundary 
conditions, both at $y=0$, listed above,

$$
\left[\begin{array}{ll}
M_{11} & M_{12} \\
M_{21} & M_{22}
\end{array}\right]\left\{\begin{array}{l}
A^{\prime} \\
A^{\prime \prime}
\end{array}\right\}=\left\{\begin{array}{l}
0 \\
0
\end{array}\right\},
$$

where

$$
\begin{aligned}
& M_{11}=\left(\frac{B}{A}\right)^{\prime}, \\
& M_{12}=\left(\frac{B}{A}\right)^{\prime \prime} \text {, } \\
& M_{21}=-b^{\prime}\left\{D_{33}+\left(\frac{B}{A}\right)^{\prime} D_{23}\right. \\
& \left.+\mu\left[D_{23}+\left(\frac{B}{A}\right)^{\prime} D_{22}\right]\right\} \\
& +\mathrm{i} k\left\{D_{13}+\left(\frac{B}{A}\right)^{\prime} D_{33}\right. \\
& \left.+\mu\left[D_{12}+\left(\frac{B}{A}\right)^{\prime} D_{23}\right]\right\} \text {, } \\
& M_{22}=-b^{\prime \prime}\left\{D_{33}+\left(\frac{B}{A}\right)^{\prime \prime} D_{23}\right. \\
& \left.+\mu\left[D_{23}+\left(\frac{B}{A}\right)^{\prime \prime} D_{22}\right]\right\} \\
& +\mathrm{i} k\left\{D_{13}+\left(\frac{B}{A}\right)^{\prime \prime} D_{33}\right. \\
& \left.+\mu\left[D_{12}+\left(\frac{B}{A}\right)^{\prime \prime} D_{23}\right]\right\} \text {. }
\end{aligned}
$$

This second eigenvalue problem is, however, nonsymmetric, putting in evidence the nonassociated character of Coulomb's friction law. The determinant of the coefficient matrix in 16 is found symbolically in terms of $c$ and $\mu$, and is composed of a real and an imaginary part. For each value of $\mu$, the characteristic equation of 16 is solved numerically in the Argand plane for $c$.

Figure 6 shows how the complex nondimensional wave velocity $c / c_{T}$ depends on the coefficient of friction for a typical set of parameters. Recall from (12) that $\operatorname{Im}(c)>0$ corresponds to an exponentially growing solution; since $\operatorname{Re}(c) \neq 0$, the solution represents an oscillation in time. In order to make the numerical verification by the interested reader possible, Table 1 shows the numerical values of the coordinates of some points of the curves in Fig. 6 .

For another set of elastic parameters, Fig. 7 shows three curves, now for three different orientations $\theta$ of the

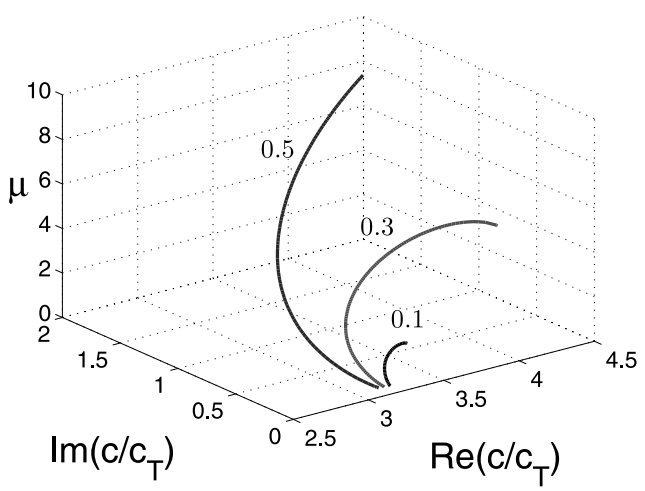

Fig. 6. Coefficient of friction $\mu$ versus the real and imaginary parts of $c / c_{T}$, for some values of Poisson's ratio $\nu_{12}$ indicated near each curve. Data: $\theta=90^{\circ}, E_{1} / E_{2}=8$, $G_{12} / E_{2}=0.1$, and $\nu=0.3$.

Table 1. Numerical coordinates of points in the three curves in Fig. 6 Data: $\theta=90^{\circ}, E_{1} / E_{2}=8, G_{12} / E_{2}=0.1$, and $\nu=0.3$.

\begin{tabular}{|c|ccc|}
\hline$\nu_{12}$ & $\mu$ & $\operatorname{Re}\left(c / c_{T}\right)$ & $\operatorname{Im}\left(c / c_{T}\right)$ \\
\hline \hline \multirow{3}{*}{0.1} & 0.4 & 3.28 & $6.32 \cdot 10^{-4}$ \\
\cline { 2 - 4 } & 2.15 & 3.38 & 0.142 \\
\cline { 2 - 4 } & 2.92 & 3.51 & $1.58 \cdot 10^{-4}$ \\
\hline \multirow{3}{*}{0.3} & 0.425 & 3.23 & $1.58 \cdot 10^{-4}$ \\
\cline { 2 - 4 } & 7.45 & 3.86 & 0.968 \\
\cline { 2 - 4 } & 10 & 4.39 & 0.802 \\
\hline \multirow{2}{*}{0.5} & 0.453 & 3.18 & $1.58 \cdot 10^{-4}$ \\
\cline { 2 - 4 } & 10 & 3.15 & 1.99 \\
\hline
\end{tabular}

principal directions of orthotropy, also illustrating how the coefficient of friction at the onset of flutter instability relates with the normalized complex wave velocity $c / c_{T}$ : in a manner similar to what is observed in Fig. 6, each curve forms a smooth arch beginning and ending in plane $\operatorname{Im}\left(c / c_{T}\right)$. Table 2 complements Fig. 7 with numerical data. Certain combinations of material constants and skew angle $\theta$ lead to coefficients of friction at the onset of flutter instability that are considerably small, as shown in the sets \{Fig. 6 Table 1 $\}$ and \{Fig. 7 Table 2\} .

Besides from determining rigorously the regions in the parameter space corresponding to unstable behaviours, it is interesting to observe the form of those unstable solutions. Figure 8 illustrates the real parts of the horizontal (a) and vertical (b) components of displacement field represented at the nondimensional instant $T=$ $\left(c_{T} / \Lambda\right) t$ (with $c_{T}=\sqrt{G_{12} / \varrho}$ and $\Lambda=1$ being an arbitrary wavelength) for $\mu=0.5$ and a given set of material data. In Fig. 8 a) there is clearly visible (i) the (exponential) decay of the $U$ component of the displacement with the depth $Y$ and (ii) the periodic (sinusoidal) form of the wave in the $X$ direction. In parallel, Fig. 8(b) shows (i) the kinematic boundary 


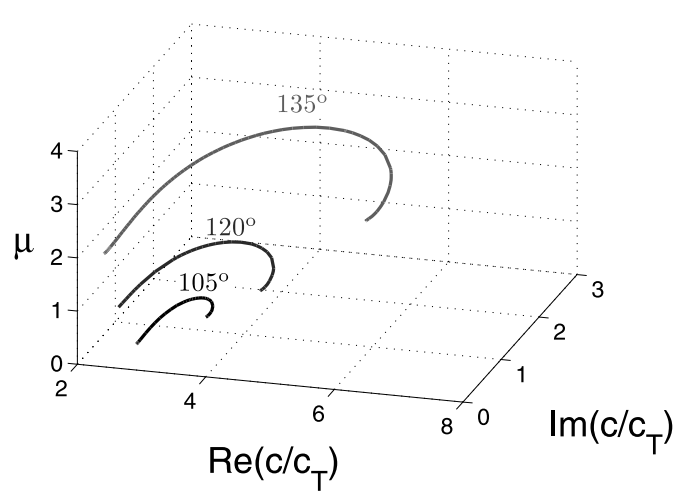

Fig. 7. Coefficient of friction $\mu$ versus the real and imaginary parts of $c / c_{T}$, for some values of the angle $\theta$ that the principal directions of orthotropy make with the sliding direction (in degrees, indicated near each curve). Data: $E_{1} / E_{2}=4, G_{12} / E_{2}=0.1, \nu=0.4$, and $\nu_{12}=0.1$.

Table 2. Some numerical solutions of the set of characteristic equations (14) and 16 for the data $E_{1} / E_{2}=4$, $G_{12} / E_{2}=0.1, \nu=0.4$, and $\nu_{12}=0.1$ (Fig.7).

\begin{tabular}{|c|ccc|}
\hline$\theta$ & $\mu$ & $\operatorname{Re}\left(c / c_{T}\right)$ & $\operatorname{Im}\left(c / c_{T}\right)$ \\
\hline \hline \multirow{3}{*}{$105^{\circ}$} & 0.483 & 2.92 & $1.58 \cdot 10^{-4}$ \\
\cline { 2 - 4 } & 0.96 & 3.47 & 0.55 \\
\cline { 2 - 4 } & 1.12 & 4 & $1.58 \cdot 10^{-4}$ \\
\hline \multirow{3}{*}{$120^{\circ}$} & 1.04 & 2.58 & $10^{-4}$ \\
\cline { 2 - 4 } & 1.6 & 3.73 & 1.13 \\
\cline { 2 - 4 } & 1.7 & 4.83 & $1.58 \cdot 10^{-4}$ \\
\hline \multirow{3}{*}{$135^{\circ}$} & 2.13 & 2.41 & $1.58 \cdot 10^{-4}$ \\
\cline { 2 - 4 } & 3.1 & 4.49 & 2.07 \\
\cline { 2 - 4 } & 3.21 & 6.48 & $1.58 \cdot 10^{-4}$ \\
\hline
\end{tabular}

condition enforcing that at the contact surface $Y=0$ there is no normal displacement $V$ and (ii) the periodic (sinusoidal) pattern of the vertical displacement $V$ in the tangent direction $X$; if a larger range of values of $Y$ was chosen to plot Fig. 8(b), the exponential decay of $V$ with depth $Y$ would also become apparent. The features exhibited in Fig. 8 are common to other material parameters leading to flutter instability.

\section{Conclusions}

It was shown that automatic symbolic computation is a valuable resource in establishing the conditions for the occurrence of divergence or flutter instabilities in orthotropic solids subjected to frictional contact conditions. In particular, symbolic computation opened the possibility of consideration of orthotropic materials in the search for the conditions of occurrence of divergence and flutter instabilities in elastic solids acted by a rough surface. By means of symbolic computation it was put in evidence that unstable solutions may exist for coefficients

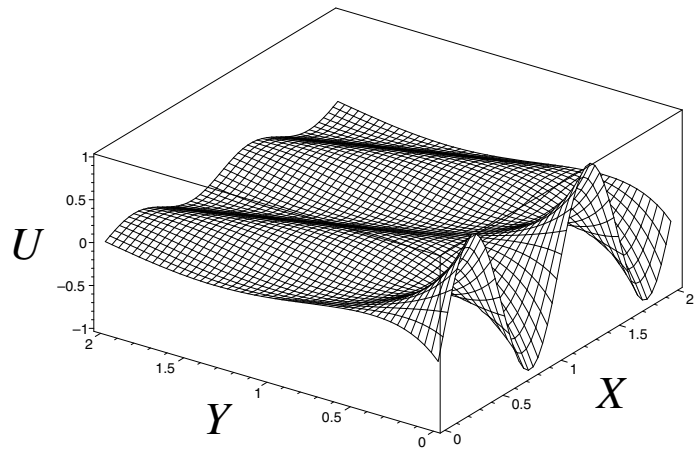

(a)

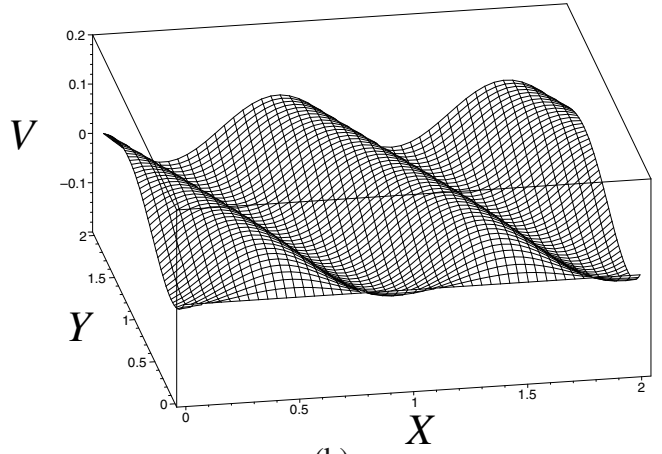

(b)

Fig. 8. Representation of an exponentially growing wave at non-dimensional time $T=0.1$ of the square $(X, Y)=$ $(x / \Lambda, y / \Lambda) \in[0,2] \times[0,2]$ near the frictional contact surface $Y=0 . \quad k=2 \pi$. Displacement parallel (perpendicular) to the frictional interface (a), displacement perpendicular to the frictional interface (b). The non-dimensional velocity $c / c_{T}=3.1380863+$ $0.0140721 \mathrm{i}$, corresponding to $E_{1} / E_{2}=8, \nu_{12}=$ $0.3, \nu=0.2, G_{12} / E_{2}=0.1, \theta=90^{\circ}, \mu=0.5$.

of friction that are much smaller than in the case of isotropic materials.

\section{Acknowledgment}

M.A. Agwa wishes to thank Fundação para a Ciência e a Tecnologia (FCT) for the scholarship SFRH/BD/31173/2006.

\section{References}

Adams, G. (1995). Self-excited oscillations of two elastic half-spaces sliding with a constant coefficient of friction, ASME, Journal of Applied Mechanics 62(4): 867-872.

Agwa, M. and Pinto da Costa, A. (2008). Instability of frictional contact states in infinite layers, European Journal of Mechanics, A: Solids 27(3): 487-503.

Agwa, M. and Pinto da Costa, A. (2011). Surface instabilities in linear orthotropic half-spaces with a frictional interface, ASME, Journal of Applied Mechanics 78(4), Paper 041002. 
Batoz, J.-L. and Dhatt, G. (1990). Modélization des Structures par Éléments Finis. Vol. 1: solides élastiques, Hermès, Paris.

Ibrahim, R. (1994). Friction-induced vibration, chatter, squeal, and chaos, Part I: Mechanics of contact and friction, Part II: Dynamics and modeling, ASME, Applied Mechanics Reviews 47(7): 209-253.

Maple (2013). Maplesoft software company, http: / /www.maplesoft.com/.

Martins, J., Faria, L. and Guimarães, J. (1992). Dynamic surface solutions in linear elasticity with frictional boundary conditions, in R. Ibrahim and A.A. Soom (Eds.), FrictionInduced Vibration, Chatter, Squeal and Chaos, ASME, New York, NY, pp. 33-39.

Martins, J., Guimarães, J. and Faria, L. (1995). Dynamic surface solutions in linear elasticity and viscoelasticity with frictional boundary conditions, ASME, Journal of Vibration and Acoustics 117(4): 445-451.

Martins, J. and Raous, M. (Eds.) (2002). Friction and Instabilities, Springer, Vienna.

Pinto da Costa, A. and Agwa, M. (2009). Frictional instabilities in orthotropic hollow cylinders, Computers and Structures 87(21-22): 1275-1286.

Rand, O. and Rovenski, V. (2005). Analytical Methods in Anisotropic Elasticity with Symbolic Computational Tools, Birkhauser, Basel.

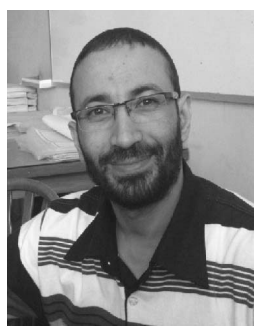

Mohamed A. Agwa was born in Bahnabay, Zagazig, Egypt, in 1974. He received his B.Sc. degree in mechanical engineering in 1997, and his M.Sc. degree in mechanical design and production engineering in 2004, both from Zagazig University, and his Ph.D. degree in engineering sciences in 2011 from Instituto Superior Técnico, Universidade Técnica de Lisboa, Portugal. Currently, he is an assistant professor at the Department of Mechanical Design and Production Engineering, Zagazig University. His research interests include modelling and numerical computation of dynamic instabilities and multiple solutions in frictional contact mechanics in the framework of finite element techniques, modelling and simulation of severe plastic deformation processes.

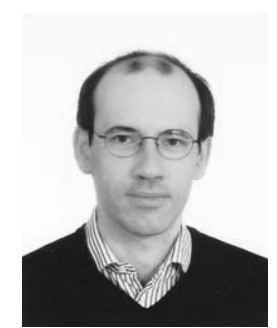

António Pinto da Costa received a B.Sc. in civil engineering in 1989, an M.Sc. in engineering of structures in 1992 and a Ph.D. in civil engineering in 2002, from Instituto Superior Técnico (Universidade Técnica de Lisboa, Portugal). Currently he serves as an assistant professor at the Department of Civil Engineering, Architecture and Georesources at the Alameda campus of Instituto Superior Técnico, Lisboa. He has taught various courses in structural mechanics and structural dynamics. His research interests include dynamic instabilities and the occurrence of bifurcations in frictional contact systems, unilateral contact problems, complementarity problems and cable dynamics.

\section{Appendix}

\section{MAPLE commands}

Tables A1 and A2 contain the main Maple commands used in the symbolic manipulation of the expressions for the study of surface divergence and flutter instabilities. In both cases the commands with(student), with (LinearAlgebra), with(DETools) and with (plots) calling relevant Maple packages were executed at the beginning of both Maple files.

Table A1. Maple commands for the study of divergence instability (Section 2).

\begin{tabular}{|c|c|}
\hline Resolution of Eqn. (1). & dsolve (Eq1, symbolic) : \\
\hline $\begin{array}{l}\text { Differentiation of the } \\
\text { expression } \mu(\lambda)(4) \text {. }\end{array}$ & $\operatorname{dmu}($ lambda $):=\operatorname{diff}(m u($ lambda), lambda) : \\
\hline $\begin{array}{l}\text { Check the vertical slopes } \\
\text { at the onset of instability. }\end{array}$ & $\operatorname{limit}(\operatorname{dmu}(\operatorname{lambda}), \operatorname{lambda}=0) ;$ \\
\hline $\begin{array}{l}\text { Numerical resolution } \\
\text { of Eqn. (4). }\end{array}$ & $\begin{array}{l}\text { for } \mathrm{k} \text { from } 0 \text { to } 400 \\
\text { do } \\
\mathrm{mu}:=\mathrm{k} / 80.0: \\
E Q 4: \\
\quad \mathrm{Y}[\mathrm{k}]:=\mathrm{fsolve}(\mathrm{EQ4}=0.0,1 \text { ambda }, 0 \ldots 100) \text { : } \\
\text { od: }\end{array}$ \\
\hline $\begin{array}{l}\text { Write the pairs }(\mu, \lambda) \\
\text { in the file "filename.txt" } \\
\text { in order to produce the graphs } \\
\text { in Fig. 2. }\end{array}$ & 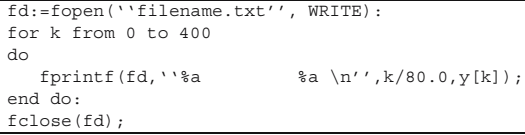 \\
\hline $\begin{array}{l}\text { Explicit expressions (6) } \\
\text { of the critical value of } \mu \text { (at the } \\
\text { onset of divergence instability). }\end{array}$ & $\operatorname{limit}(\mathrm{mu}(\mathrm{lambda}), \operatorname{lambda}=0)$; \\
\hline
\end{tabular}

Table A2. Maple commands for the study of flutter instability (Section 3).

\begin{tabular}{|c|c|}
\hline Solution of Eqn. (1). & dsolve (Eq1, symbolic) : \\
\hline $\begin{array}{l}\text { Symbolic computation of the } \\
\text { determinants in Eqns. (13) and (16). }\end{array}$ & $\operatorname{det}($ Coeff_Matrix); \\
\hline $\begin{array}{l}\text { To gather the terms of the } \\
\text { same power of } b \text {. }\end{array}$ & collect (det (Coeff_Matrix), b) ; \\
\hline $\begin{array}{l}\text { Solution of Eqn. (14) } \\
\text { to find its four solutions. }\end{array}$ & $\begin{array}{l}\text { Solve }(\{\text { Eq14 }=0\},\{b\}) ; \\
\text { allvalues }(b) ;\end{array}$ \\
\hline $\begin{array}{l}\text { To solve the characteristic } \\
\text { equation in Eqn. (16) to find } \\
\text { the values of } c \text { in the Argand } \\
\text { plane for prescribed values of } \mu \text {, } \\
\text { to draw Figs. } 6 \text { and } 7 \text {. }\end{array}$ & $\begin{array}{l}\text { for } k \text { from } 1 \text { to } 400 \\
\text { do } \\
\text { mu: }:=k / 40: \\
\text { DD: }:=\operatorname{det}(\text { Coeff_Matrix_Eq16): } \\
\quad y[k]:=\text { fsolve }(\overline{D D}=0.0, \text { c complex }, 0 \ldots 5.0+5.0 * I) \text { : } \\
\text { od: }\end{array}$ \\
\hline
\end{tabular}

Received: 11 October 2013

Revised: 22 July 2014

Re-revised: 25 October 2014 